\title{
Silicone Implant Sandwiched between Intact Nasal Bones with Fractured Nasal Bone Segments
}

\author{
Soo Hyun Woo, \\ Woo Seob Kim, \\ Han Koo Kim, \\ Tae Hui Bae
}

Department of Plastic and Reconstructive Surgery, Chung-Ang University Hospital, Chung-Ang University College of Medicine, Seoul, Korea

No potential conflict of interest relevant to this article was reported.

\begin{abstract}
As the number of people who have undergone augmentation rhinoplasty has increased recently, nasal fractures are becoming more common after rhinoplasty. A silicone implant can affect the nasal fracture pattern, but there is no significant difference in treatment methods commonly. A 28-year-old female who had undergone augmentation visited our clinic with a nasal fracture. Computed tomography revealed that the silicone implant was sandwiched between the intact nasal bones with fractured bone fragments. In this case, open reduction was inevitable and a new silicone implant was inserted after reduction. Migration of the silicone implant beneath the nasal bone is a very rare phenomenon, but its accurate prevention and diagnosis is important because a closed reduction is impossible.
\end{abstract}

Keywords: Nasal bone / Rhinoplasty / Silicones

\section{INTRODUCTION}

A nasal bone fracture is the most common facial bone fracture encountered by plastic surgeons. The number of augmentation rhinoplasty procedures performed for cosmetic purposes has increased significantly in Korea and other Asian countries. Accordingly, cases of nasal bone fracture among patients who underwent augmentation rhinoplasty using silicone implants have been reported. Even small nasal bone fractures can give rise to cosmetic problems and require reduction, which in most cases is closed reduction $[1,2]$. When nasal bone fractures occur in patients with silicone implants, the implants may affect fracture patterns; however, they usually do not alter the method of treatment except in severe fractures. Jeon et al. [2] reported a new classification of nasal bone fractures with silicone implant and found that the upper half of a nasal fracture is the most common fracture type that has a silicone implant. Closed reduction is enough at this time. Not

Correspondence: Woo Seob Kim

Department of Plastic and Reconstructive Surgery, Chung-Ang University Hospital, ChungAng University College of Medicine, 102 Heuksuk-ro, Dongjak-gu, Seoul 06973, Korea

E-mail:kimws@cau.ac.kr

Received December 2, 2016 / Revised January 25, 2017 / Accepted January 26, 2017 only a fracture pattern, but also other complications can be affected by silicone implants such as discoloration, infection, extrusion, and contractive distortion [3]. Displacement of nasal implant is a common complication of augmentation rhinoplasty, but migration under the nasal bone is extremely rare. Only one case has been reported where the silicone implant has slipped back to the nasal turbinate [4]. Herein, we report a rare case in which a silicone implant became sandwiched between fractured nasal bone segments, for which open reduction was inevitably performed. This is an important issue that cannot be overlooked as a potential complication for a patient who has a silicone implant on the nose.

\section{CASE REPORT}

A 28-year-old female patient visited the clinic due to nasal swelling after being punched. The patient had undergone augmentation rhinoplasty using a silicone implant in a local clinic six years prior, and had no history of other plastic procedures. Irregular palpations occurred due to the silicone implant. Radiography (both nasal lateral view and skull Waters' view) and facial bone 
3-dimensional computed tomography were performed. Based on the results, the patient was diagnosed with nasal bone fracture. However, the cephalic end of the implant was sandwiched between the intact nasal bone and the fractured nasal bone segments (Fig. 1), making closed reduction impossible. Open reduction was necessary to remove the silicone implant and set the fractured bones. Therefore, an open approach was attempted by performing an inverted- $\mathrm{V}$ columella incision while the patient was under general anesthesia. Dissection was made from the nasal cartilage up to the nasal radix, and the sandwiched implant was identified. A fracture line could be clearly seen after the implant was removed (Fig. 2). Open reduction was performed under visual inspection, and a new implant was inserted up to the nasal radix at the patient's request (Fig. 3). No problems or complications arose during the surgery. The nasal reconstruction was successful with no sign of infection, contour change, or migration of implant during a year of follow-up.

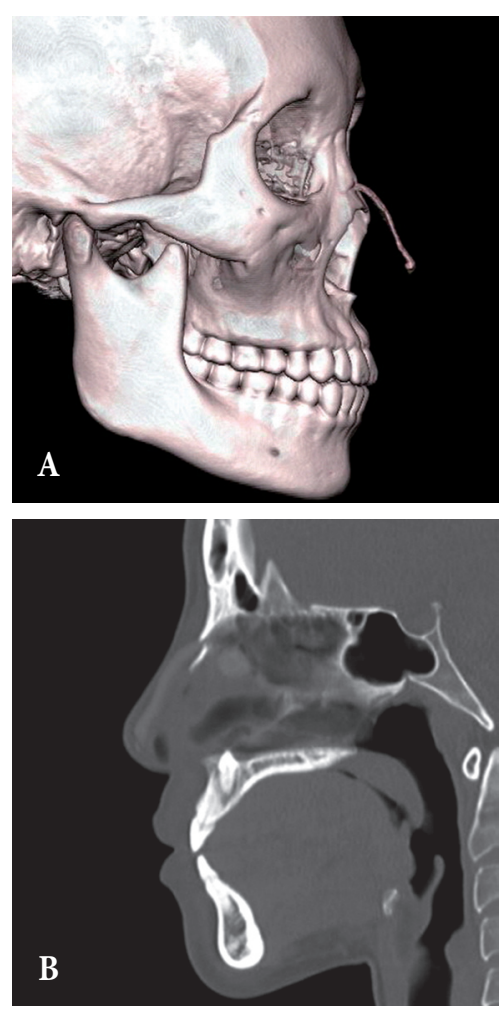

Fig. 1. Computed tomography findings before surgery. A silicone implant is sandwiched between the fractured nasal bone segments. The caudal end of the silicone implant has reached the nasal tip. Three-dimensional image (A) and sagittal image (B).

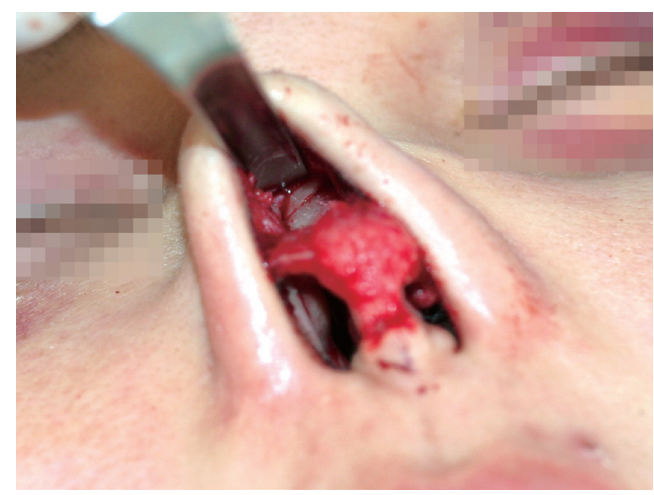

Fig. 2. Appearance during surgery. A fracture line was observed after the implant sandwiched between the fractured nasal bone segments was removed.
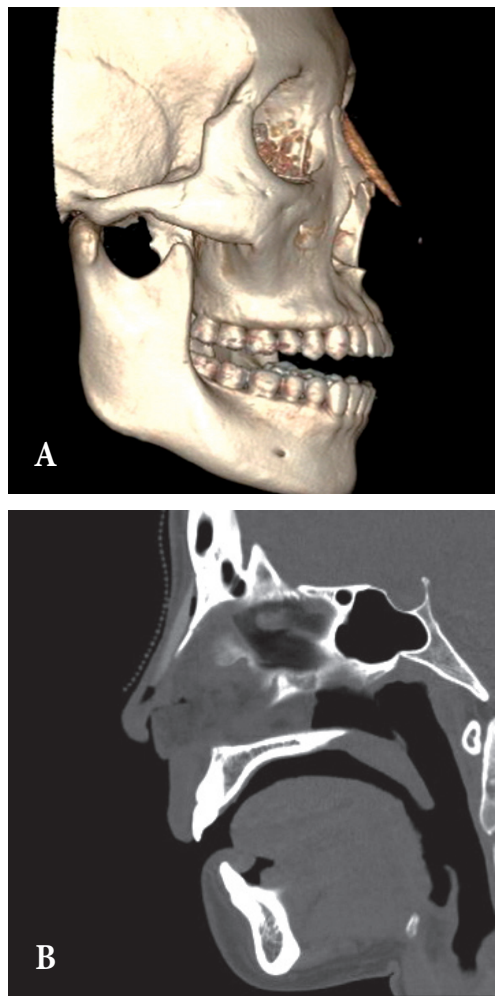

Fig. 3. Computed tomography findings after surgery. The nasal bones were set back in their original places. The new implant was inserted up to the nasion. Three-dimensional images (A), and sagittal images (B).

\section{DISCUSSION}

A nasal fracture is not an exception for those who undergo augmentation rhinoplasty for cosmetic purposes. The differences in management of a nasal bone fracture with and without an im- 
plant are determined by the differences in both diagnosis and treatment. Hwang et al. [5] reported that nasal bone fractures in the absence of implants can be examined using plain radiographs in $82 \%$ of cases. However, access to the fractured nasal bone segments may be difficult in the presence of silicone implants. For this reason, it is difficult to make a diagnosis based on physical examination only. Since implants also interfere with clear X-ray images, computed tomography must be performed in patients with silicone implants who are suspected of having a nasal bone fracture [2]. Although numerous studies have been conducted on the treatment of common nasal bone fractures, research on the treatment of nasal bone fractures in patients with silicone implants is lacking. Jeon et al. proposed a classification method that divides nasal bone fractures in patients with silicone implants into three types. They also suggested appropriate treatment methods for each type of fracture, and explained that silicone implants had to be removed only when the supporting structure had been damaged [2]. However, the case in this study was an exception, as the implant had to be removed regardless of the fracture type.

In the present case, a new implant was inserted immediately after fracture reduction. In the past, performing fracture reduction in conjunction with implant insertion was not recommended due to the instability of the fractured area and possible resorption of nasal bone [6]. However, the nasal bone fracture was not accompanied by a septal fracture, and nasal bone reduction and implant insertion were performed together in the present case so that the patient's cosmetic requests could be managed without the need for another surgery in the future. The results were highly satisfactory.

Nasal bone flattening due to the silicone implant and bone erosion commonly occur among patients who undergo augmentation rhinoplasty; as a result, these patients are at a higher risk of nasal bone fracture than those who did not undergo augmentation rhinoplasty [3]. Therefore, when the increase in rhinoplasty is taken into consideration, in a similar case such as the present case, this is likely to occur again.

In the present case, it appears that the silicone implant had not been properly inserted from the nasal tip up to the radix due to its insufficient length; as a result, it ended up being sandwiched be- tween the fractured nasal bone segments. For this reason, it was not possible to perform closed reduction. Instead, open reduction had to be performed along with replacement or removal of the implant. This procedure can have unfavorable outcomes in terms of cost, and may put great pressure on the surgeon as an incision must be performed prior to the procedure; therefore, prevention is always recommended. While autologous cartilage is now commonly used in tip augmentation procedures, it was previously more common to use L-shaped implants, or insert a linear-shaped implant all the way down to the nasal tip in the past. In the latter case, the implant may not reach the nasal radix due to insufficient length on rare occasions, as was the case for the patient in this study.

Migration of silicone implant under the nasal bones is an unusual phenomenon that can only occur when a patient with a fracture has a silicone implant. This is important because it is a deadly phenomenon where closed reduction is impossible. When we encounter nasal trauma in a patient who has undergone augmentation rhinoplasty, surgeons should be aware that the nasal implant can migrate under the nasal bone and computed tomography scan is essential when diagnosing a fracture.

\section{REFERENCES}

1. Chen CT, Hu TL, Lai JB, Chen YC, Chen YR. Reconstruction of traumatic nasal deformity in Orientals. J Plast Reconstr Aesthet Surg 2010;63:257-64.

2. Jeon SP, Kang SJ, Kim JW, Kim YH, Sun H. New nasal fracture classification for patients with silicone implants. J Plast Surg Hand Surg 2013;47:363-7.

3. Jung DH, Kim BR, Choi JY, Rho YS, Park HJ, Han WW. Gross and pathologic analysis of long-term silicone implants inserted into the human body for augmentation rhinoplasty: 221 revision cases. Plast Reconstr Surg 2007;120:1997-2003.

4. Lin HT, Day YJ, Sum DC, Liu FC, Liou JT. Displaced nasal silicone implant: an unusual cause of nasotracheal tube obstruction. J Clin Anesth 2013;25:344-5.

5. Hwang K, You SH, Kim SG, Lee SI. Analysis of nasal bone fractures; a six-year study of 503 patients. J Craniofac Surg 2006;17:261-4.

6. Kim JH, Lee JW, Park CH. Cosmetic rhinoseptoplasty in acute nasal bone fracture. Otolaryngol Head Neck Surg 2013;149:212-8. 